\title{
Influence of carbon nanotube clustering on the electrical conductivity of polymer composite films
}

\author{
J. O. Aguilar ${ }^{1,2}$, J. R. Bautista-Quijano ${ }^{1}, F$. Avilés ${ }^{1 *}$ \\ ${ }^{1}$ Centro de Investigación Científica de Yucatán, Unidad de Materiales, Calle 43 No. 130 Col. Chuburná de Hidalgo, \\ 97200, Mérida Yucatán, México \\ 2Universidad de Quintana Roo, División de Ciencias e Ingeniería, Boulevard Bahía s/n esq. Ignacio Comonfort, \\ Col. Del Bosque, 77019 Chetumal Quintana Roo, México
}

Received 6 January 2010; accepted in revised form 9 March 2010

\begin{abstract}
Electrical conductivity of $150-200 \mu \mathrm{m}$ thick polysulfone films loaded with $0.05-0.75 \%$ w/w multiwall carbon nanotubes was systematically investigated for two types of dispersion states, uniformly dispersed and agglomerated at the micro-scale. The percolation threshold was found at $0.11 \%$ and $0.068 \% \mathrm{w} / \mathrm{w}$ for the uniformly dispersed and agglomerated films, respectively. Overall, the conductivity of the films with agglomerated nanotubes was higher than that of the uniformly dispersed ones, with marked differences of 2 to 4 orders of magnitude for carbon nanotubes loadings in the upper vicinity of the percolation threshold $(0.1-0.3 \% \mathrm{w} / \mathrm{w})$. The increased conductivity of the agglomerated state is explained by the increased nanotube-to-nanotube contact after the percolating network has formed, which facilitates electron transfer.
\end{abstract}

Keywords: polymer composites, electrical conductivity, MWCNT, percolation, clustering

\section{Introduction}

Carbon nanotube (CNT)-polymer composites have been aggressively investigated for more than a decade, given the extraordinary physical properties of CNTs and the versatility of polymeric materials. In recent years, a great deal of attention has been paid to the electrical properties of such composites, given the great promises that these materials hold as multifunctional materials in the area of electronics, sensors, and actuators [1-4]. Electrical conductivity of CNT-polymer nanocomposites using very low CNT weight loadings typically reaches the level of semiconductors $(\sim 0.001-0.1 \mathrm{~S} / \mathrm{m})$ [4-7]. The conductivity of these composites can reach up to several hundreds of $\mathrm{S} / \mathrm{m}$ when CNTs are aligned or decorated $[8,9]$. The critical CNT content required to form a percolation network depends mainly on the CNT type (single-wall carbon nanotube, SWCNT, or multi-wall carbon nanotube, MWCNT), intrinsic CNT quality (amorphous carbon content and ratio metallic/semi-conductive tubes), aspect ratio $(L / d)$, morphology, polymer matrix and dispersion state, and hence the range of reported percolation thresholds for CNT-polymer systems is vast, see e.g. [4-11]. For the same polymer and CNT intrinsic quality, dispersion state and CNT aspect ratio have been recognized as the critical factors governing composite conductivity [11]. Using an epoxy-MWCNT system, Li et al. [11] found that when the CNT aspect ratio is larger than $\sim 100$, dispersion becomes the main factor controlling electrical percolation. Conventionally, it is accepted that a good CNT dispersion within the polymer enhances the physical properties of the composite [12, 13]. However, a few studies suggest that CNT agglomeration could favor the formation of a percolating network $[11,14,15]$. The great majority of these studies, however, are related to 
bulk geometry, while the thin film configuration has been scarcely investigated. Conductive CNT composites in film geometry show a great potential in micro- and nano-electronics, micro-electromechanical systems (MEMS), nano-electromechanical systems (NEMS), and photoelectronics, among many others [16]. We report in this work compelling experimental evidence that shows that MWCNT agglomeration at the micro-scale significantly favors electrical conductivity of CNT-polymer composite films, especially for weight loadings in the upper vicinity of the percolation threshold.

\section{Experimental}

\subsection{Synthesis of PSF-CNT films}

Multiwall carbon nanotubes grown by chemical vapor deposition were supplied by Bayer Material Science ${ }^{1}$ ('Baytubes C150P ${ }^{\circledR}$ '). The nanotubes have average inner and outer diameters of $\sim 4$ and $\sim 13 \mathrm{~nm}$, respectively, and length of $1-4 \mu \mathrm{m}$. SEM images of the MWCNTs employed can be found in a previous work [17]. Commercial polysulfone, PSF (UDEL P-1700 ${ }^{\circledR}$, volume resistivity = $3 \cdot 10^{16} \Omega \cdot \mathrm{cm}$ ), obtained from Solvay Advanced Polymers $^{2}$ was used to fabricate the polymer films. Prior to film fabrication PSF was dried in a convection oven at $150^{\circ} \mathrm{C}$ for $3.5 \mathrm{~h}$. For film fabrication, approximately $2 \mathrm{~g}$ of PSF was dissolved in $10 \mathrm{ml}$ of chloroform and stirred for 2 hours. Separately, a range of weight fractions $(0.05-0.75 \% \mathrm{w} / \mathrm{w})$ of the as-received MWCNTs were dispersed in chloroform using a conventional ultrasonic bath for 3-11 hours, depending on the MWCNT content. Details of solvent content and sonication time employed to disperse the CNTs in chloroform are listed in Table 1. The long sonicating times employed promote disentanglement of the CNT bundles at the nanoscale, see e.g. [11, 18]. Both solutions were then mixed and mechanically stirred for $30 \mathrm{~min}$. The polymer/chloroform/CNT solution was then sonicated for $1 \mathrm{~h}$ and finally stirred again for 10 min prior to casting the viscous solution. Polymer films were cast onto a Petri dish and covered to slow the solvent evaporation rate, to form an homogeneous, well dispersed film with a uni-

${ }^{1}$ Bayer Material Science, Leverkusen, Germany.

www.baytubes.com

${ }^{2}$ Solvay Advanced Polymers, LLC, Alpharetta, Georgia. www.solvayadvancedpolymers.com
Table 1. Amount of solvent and sonication time employed for composite film fabrication

\begin{tabular}{|c|c|c|c|}
\hline$\%$ CNT & $\begin{array}{c}\text { MWCNTS } \\
{[\mathbf{m g}]}\end{array}$ & $\begin{array}{c}\text { Chloroform } \\
{[\mathbf{m l}]}\end{array}$ & $\begin{array}{c}\text { Sonication } \\
\text { time }[\mathbf{h}]\end{array}$ \\
\hline 0.050 & 1.0 & 7 & 3 \\
\hline 0.075 & 1.5 & 7 & 3 \\
\hline 0.100 & 2.0 & 10 & 5 \\
\hline 0.200 & 4.0 & 13 & 7 \\
\hline 0.300 & 6.0 & 15 & 9 \\
\hline 0.500 & 10.0 & 20 & 11 \\
\hline 0.750 & 15.0 & 20 & 11 \\
\hline
\end{tabular}

form thickness of 150-200 $\mu \mathrm{m}$. The group of films fabricated in this way will be hereafter referred to as 'uniformly dispersed'. To obtain the uniformly dispersed films, about $75 \%$ wt. of the employed chloroform was evaporated prior to solution casting, which yielded a rather viscous solution during casting. For the second group of PSF-CNT films ('agglomerated') reduced viscosity of the CNT/ polymer/chloroform solution was achieved by decreasing the proportion of chloroform evaporated prior to casting to about $50 \%$ wt. This technique reduced the viscosity of the solution poured on the Petri dish and hence promoted CNT re-agglomeration right after casting. Apart from controlling the viscosity of the solution, the rest of the fabrication procedure and processing times employed for the 'agglomerated' films were identical to those used for the 'uniformly dispersed'ones. All films were dried at room temperature for 1 day after casting, and further dried in a vacuum oven at $100^{\circ} \mathrm{C}$ for 1 day. The final product was a composite film with a thickness of $\sim 150-200 \mu \mathrm{m}$ with randomly distributed MWCNTs. It is important to point out that, in the context of this work, the terms 'uniformly dispersed' and 'agglomerated' refer to a dimensional scale ranging from centimeters (macroscopic) to a few hundreds of microns. Below that dimensional scale, small clusters of CNTs are visible for both groups of films, as it will be further discussed.

\subsection{Electrical properties}

Electrical conductivity of PSF-CNT films loaded with different weight fractions $(0.05-0.75 \% \mathrm{w} / \mathrm{w})$ of MWCNTs was measured at room temperature using a Fluke electrometer ${ }^{3}$. The electrometer is capable of measuring DC electrical resistance up to

3Fluke 1550B MegOhmMeter, Fluke Corporation, Everett, Washington 

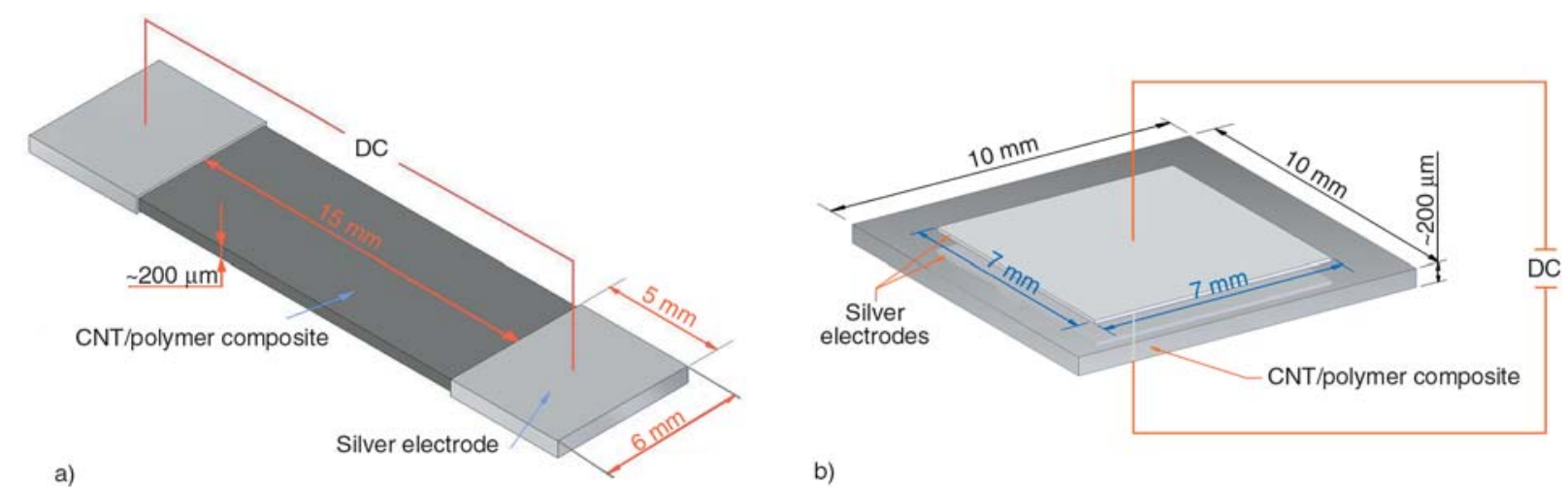

b)

Figure 1. Schematic illustrations of the test specimens employed: a) CNT loadings $>0.1 \% \mathrm{w} / \mathrm{w}$, voltage is applied through the specimen length, b) CNT loadings $0.05-0.1 \% \mathrm{w} / \mathrm{w}$, voltage is applied across the specimen thickness

$1 \mathrm{~T} \Omega$. DC volumetric resistance $(R)$ was measured using two silver paint ${ }^{4}$ electrodes of $5 \mathrm{~mm}$ length painted on the film edges of $25 \mathrm{~mm}$ long specimens, leaving an effective span $(L)$ of $15 \mathrm{~mm}$ between the silver electrodes, see Figure 1a. Specimen width was $6 \mathrm{~mm}$ and film thickness $\sim 150$ $200 \mu \mathrm{m}$. To reduce surface effects in the measurements, silver paint electrodes were painted completely covering the ends of the sample. A DC voltage was applied through the length of the specimen and the conductivity $(\sigma)$ was calculated using Equation (1):

$\sigma=\frac{L}{A R}$

where $A$ is the cross sectional area of the sample and $R$ the measured electrical resistance.

For specimens with CNT loadings $<0.1 \%$ w/w the measured resistance exceeded the capacity of the electrometer $(R>1 \mathrm{~T} \Omega)$ and a different method had to be employed for those samples. For those cases, measurements were conducted across the specimen thickness with back-to-back silver paint electrodes covering top and bottom square areas of $7 \mathrm{~mm}$ and leaving a non-painted trim of $3 \mathrm{~mm}$ along the edge of the samples, to minimize surface effects along the edges, see Figure 1b. Since the film thickness $(\sim 150-200 \mu \mathrm{m})$ is substantially larger than the largest CNT agglomerate $(\sim 20 \mu \mathrm{m})$, this configuration should yield bulk values of resistance, although small surface contributions may not be neglected. The application of a voltage through the film thickness allowed measurement of electrical resistance $(R<1 \mathrm{~T} \Omega)$, which were converted into conductivity using Equation (1) with $L$ as the film thickness and $A$ as the $7 \times 7 \mathrm{~mm}^{2}$ in-plane area. Schematic representations of both specimens are depicted in Figure 1. Voltages employed in the resistivity measurements were $1000 \mathrm{~V}$ for 0.05 to $0.1 \% \mathrm{w} / \mathrm{w}, 500 \mathrm{~V}$ for $0.2-0.3 \% \mathrm{w} / \mathrm{w}$ and $250 \mathrm{~V}$ for $0.5-0.75 \% \mathrm{w} / \mathrm{w}$. The percolation threshold was calculated using the well-known scaling law describing the statistical percolation behavior in the vicinity of the percolation limit (see Equation (2)), which refers to a situation where randomly distributed elongated rods form percolating paths:

$$
\sigma=\sigma_{0}\left(\phi-\phi_{c}\right)^{t}
$$

where $\sigma_{0}$ is a scaling factor that may be comparable to the effective conductivity of the filler $[19,20], \phi$ is the CNT weight fraction, and $\phi_{c}$ is the percolation critical concentration. The parameter $t$ is a critical exponent that governs the scaling law in the vicinity of percolation and has been associated to the dimensionality of the system, although its physical meaning is still controversial $[10,19]$. The parameters $t$ and $\phi_{c}$ were calculated by plotting $\log \sigma$ vs. $\log \left(\phi-\phi_{c}\right)$ and varying $\phi_{c}$ until the best linear fit is obtained, see e.g. [10,19].

All properties of the composite films were measured after evaporation of the solvent, i.e. over a solid film. Thus, the percolation measured is a static (statistical) one, where CNTs cannot easily move inside the matrix. It has been reported that very low percolation limits can be achieved when the matrix posses a fluid-like state of low viscosity, where particles are able to move inside the polymeric matrix [21, 22]. This phenomenon is called dynamic (or kinetic) percolation and needs to be described by dynamic colloid theory [10, 21, 22]. 


\section{Results and discussion}

The final dispersion state of CNTs within the polymer results from a competition between van der Waals interactions among the CNTs and the viscous forces acting within the polymer solution. Low viscosity facilitates dispersion during film processing but also promotes CNT re-agglomeration right after pouring the polymer in the mold, i.e., as soon as the external energy supplied for dispersion is suspended. On the other hand, high viscosity may make processing difficult but prevents CNT re-agglomeration after casting. Therefore, the dispersion state of the CNTs within the polymer film can be controlled by modifying the viscosity of the polymer/chloroform solution, as performed in this work.

The state of CNT dispersion at the macro- and micro-scales within the composite films was evalu-
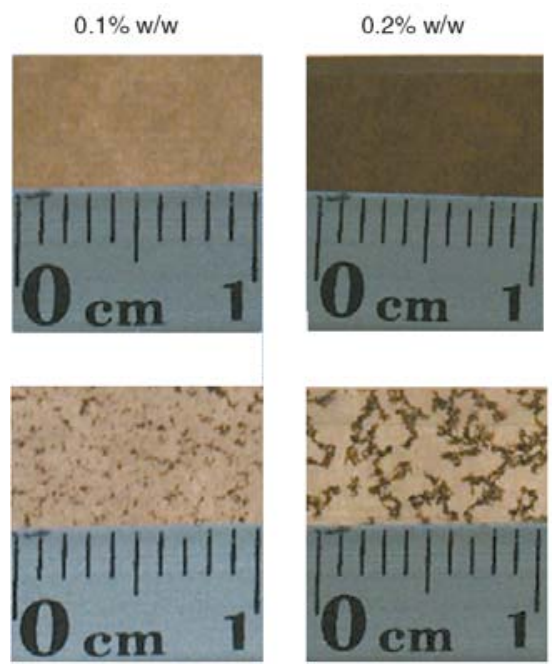

ated here using optical observations. Figure 2 shows photographs of the PSF-CNT films for selected weight fractions (columns) and the two examined dispersion states (rows), viz. uniformly dispersed (upper row) and agglomerated (lower row). Only selected weight loadings are shown in Figure 2 to depict the dispersion states. At the macro-scale, uniformly dispersed films appear as homogeneous materials with an optical transparency that decreases with increased CNT content. Agglomerated films, on the other hand, show CNT clusters with an apparent inter-cluster distance which diminishes with increased CNT content. The cluster distribution is rather uniform, with clusters in the sub-millimeter scale which tend to coalesce as the CNT concentration is increased. At this scale (millimeters), the different dispersion state of both groups of films is evident, even by naked eye.
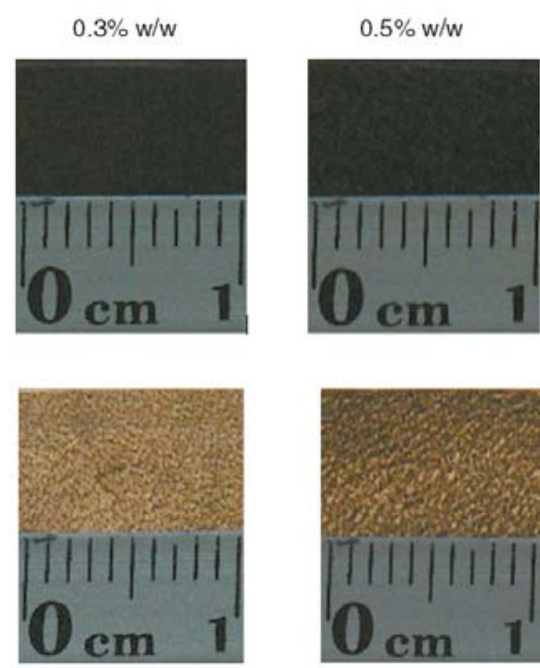

Figure 2. Millimeter-scale photographs of PSF-CNT films loaded with $0.1-0.5 \% \mathrm{w} / \mathrm{w}$ MWCNTs for two dispersion states: uniformly dispersed (upper row) and agglomerated (lower row)
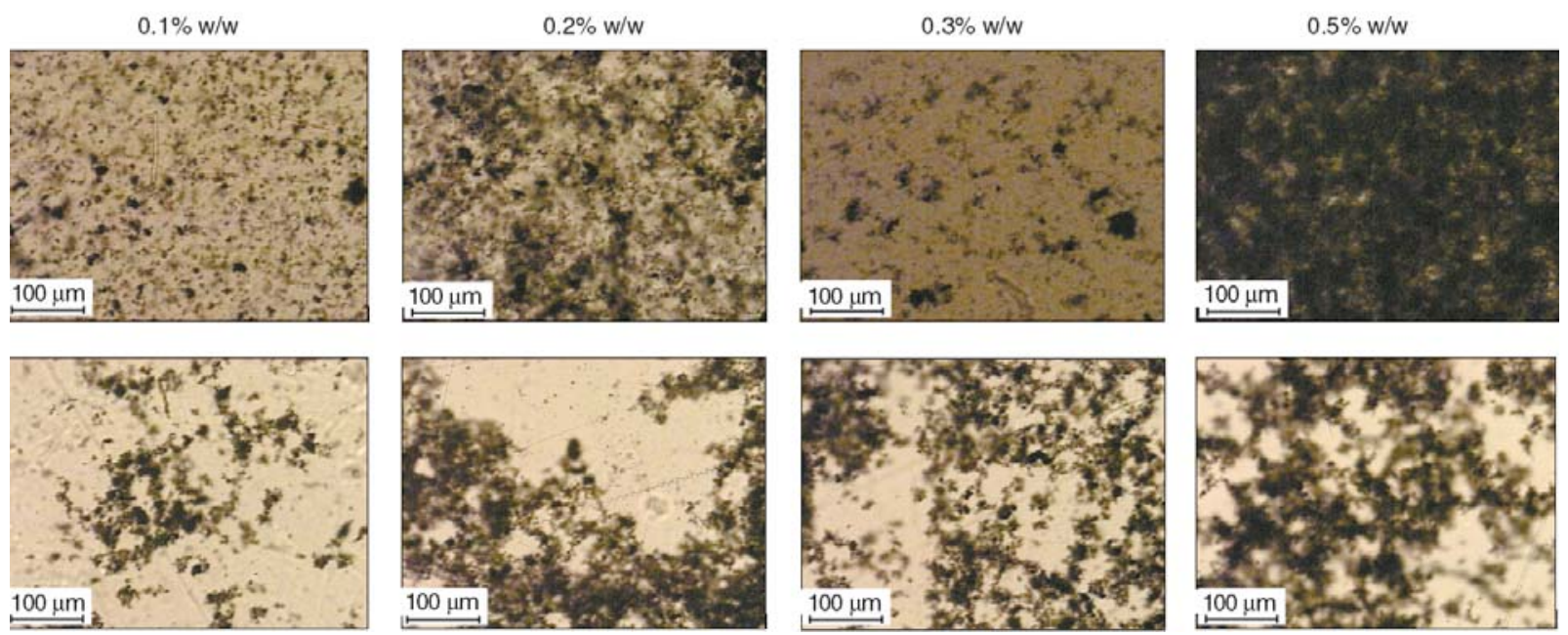

Figure 3. Optical microscopies of PSF-CNT films loaded with $0.1-0.5 \%$ w/w MWCNTs for two dispersion states: uniformly dispersed (upper row) and agglomerated (lower row) 
Figure 3 presents optical micro-scale images of films with the same weight loadings as in Figure 2. At this scale (micrometers), small CNT clusters are observed. Interestingly, even the films that appear as homogeneous and uniformly dispersed at the macro-scale, show fine clusters at the micrometer scale, but cluster size is significantly larger for the agglomerated films when compared to the uniformly dispersed ones. For example, for $0.1 \% \mathrm{w} / \mathrm{w}$ loading the individual cluster size is about $10 \mu \mathrm{m}$ for the uniformly dispersed films and about $25 \mu \mathrm{m}$ for the agglomerated ones. Cluster size increases with increased CNT loading.

As observed from these figures, agglomeration promotes CNT-to-CNT interactions through surface contact (or tunneling) and hence it should increase the electrical conductivity of the composite, as it will be further discussed. In fact, as a parallel experiment, it was observed that when the film thickness is reduced to the order of the cluster size $(\sim 20 \mu \mathrm{m})$, relatively high electrical conductivity $(\sim 0.6 \mathrm{~S} / \mathrm{m})$ is achieved through the film thickness even for films loaded with only $0.05 \% \mathrm{w} / \mathrm{w}$, which are not conductive in the in-plane (length) direction. This observation may lead to ultra-low percolation levels in the through-thickness direction of the film, especially for agglomerated films, but a detailed systematic investigation of this phenomenon falls beyond the scope of the present work. A similar phenomenon has been recently reported by Fu et al. [16], which states the percolation threshold can be reduced significantly if the film thickness is reduced to the length range of the CNTs. In that case, very low CNT loadings would be required to form a conductive path across the film thickness. In our case, the film thickness $(\sim 150-200 \mu \mathrm{m})$ is substantially larger than the CNT length $(1-4 \mu \mathrm{m})$ and CNT agglomerate size $(\sim 20 \mu \mathrm{m})$, so throughthickness conduction does not occur for low CNT weight loadings.

Figure 4 shows electrical conductivity of the composite films as a function of weight loading for uniformly dispersed and agglomerated films. Overall, the conductivity of the agglomerated films is larger than that of the uniformly dispersed ones, irrespective of the CNT loading. At low CNT loadings $(<0.1 \% \mathrm{w} / \mathrm{w})$ the film is still an isolating material and the difference in conductivity between both dispersion states is almost indistinguishable. Around $0.1 \% \mathrm{w} / \mathrm{w}$ a sharp increase in conductivity

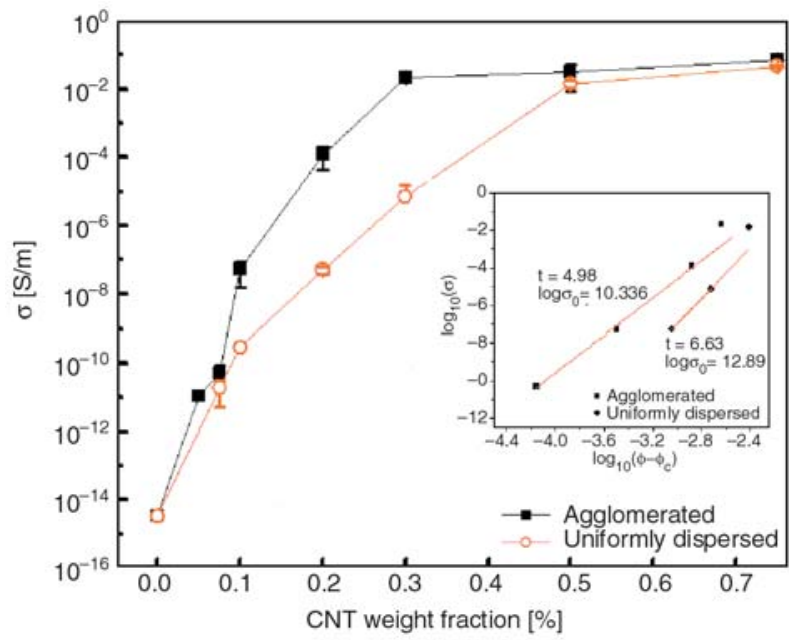

Figure 4. Electrical conductivity vs. CNT loading for uniformly dispersed and agglomerated films

is observed, indicating the formation of a percolation network. Percolation thresholds were calculated for both dispersion states fitting the measured data to the power law expression for conductivity of statistical percolation theory, Equation (2). For uniformly dispersed films, the percolation threshold $\left(\phi_{c}\right)$ and critical exponent $(t)$ were found as $0.11 \% \mathrm{w} / \mathrm{w}$ and 6.63 , respectively. On the other hand, for films with agglomerated CNTs, $\phi_{c}$ and $t$ were found as $0.068 \% \mathrm{w} / \mathrm{w}$ and 4.98 . Slightly above the percolation threshold $(0.1-0.3 \% \mathrm{w} / \mathrm{w})$, the difference in electrical conductivity between both dispersion states is large ( 2 to 4 orders of magnitude). At higher loadings ( $\geq 0.5 \% \mathrm{w} / \mathrm{w})$, the conductivity of both dispersion states is again similar, since the conductive network has been well defined and no further drastic changes are expected according to percolation theory. Notice that the aspect ratio of the employed CNTs is in the range of 80-300 and therefore the CNT dispersion state should be the most influential factor on the composite electric conductivity, according to a previous investigation by Li et al. [11].

Typical values of $t$ reported for CNT-polymer composites are in the range of 1.3-4, see [10], although a few works report values of $t$ of $~ 4.9$ [23] and even higher than 7 [24]. Mathematically, since $\phi-\phi_{c}$ is a small fraction $(<1)$, a lower value of $t$ in Equation (2) means more abrupt increments in electrical conductivity in the vicinity of percolation. The physical interpretation of the critical exponent $t$ is more complex, and still a matter of controversy, as stated in a recent review by Bauhofer and Kovacs [10]. This exponent is frequently associated to the 
system dimensionality, with values of $t \approx 1.3$ (or slightly higher) representing a two-dimensional network and $t \approx 2$ (or slightly higher) a threedimensional one $[10,16,20,25]$. However, the use of a scaling law for statistical percolation is limited to a concentration range very close to the percolation threshold and the use of this law to extract geometrical information about the CNT network from experimentally determined values of $t$ is controversial, as stated by several authors $[10,18,20]$. In our case, the relatively high values of $t$ obtained are due to the 'moderate' ( $2-4$ orders of magnitude) changes in electrical conductivity in the vicinity of percolation, which might be related to geometrical constrains imposed by the limited thickness of the film and/or to the specific polymer employed.

The higher conductivity of the films in the agglomerated state compared to the 'uniformly dispersed' ones may be related to the increased CNT-to-CNT contact/junctions in the agglomerated state. The tunneling distance allowed for electron hopping has been reported between 5-30 nm [11, 26, 27]. Agglomeration may not significantly improve the conductivity of the composite before the CNT-to$\mathrm{CNT}$ distance has reached at least this tunneling separation. Thus, the percolation threshold observed for both dispersion states investigated herein is somewhat similar. However, once the percolation network has been formed, CNT agglomeration increases the surface contact among CNTs (CNTto-CNT junctions) and hence facilitates the electrons flow through the formed CNT network. Recent studies suggest that the electrical conductivity of CNT polymer composites can be improved by increasing the number of CNT-CNT junctions per unit area, which will improve the transport of free carriers between the nanotubes [28, 29].

For higher CNT loadings the conductive network is densely packed and no further increases in electrical conductivity are expected to be caused by this mechanism. Our experimental observations are also supported by a continuum micromechanics model developed by Seidel and Lagoudas [27], which suggests that increased CNT bundling promotes the formation of conductive networks and such a networks are primarily responsible for the conductivity in MWCNT composites.

\section{Conclusions}

The influence of CNT clustering on the electrical properties of polymer composite films was investigated using two dispersion states: uniformly dispersed and agglomerated at the micro-scale. For uniformly dispersed films, the percolation threshold $\left(\phi_{c}\right)$ and critical exponent $(t)$ were found as $0.11 \% \mathrm{w} / \mathrm{w}$ and 6.63 , respectively. On the other hand, for films with agglomerated CNTs, $\phi_{c}$ and $t$ were found as $0.068 \% \mathrm{w} / \mathrm{w}$ and 4.98 . It was found that films with micrometer-size agglomerations have a slightly lower percolation threshold and higher electrical conductivity than films with uniformly dispersed MWCNTs, especially for CNT loadings in the upper vicinity of the percolation threshold $(0.1-0.3 \% \mathrm{w} / \mathrm{w})$. The increased conductivity of the agglomerated state is explained by the increased density of CNT-to-CNT junctions after the percolating network has formed, which favors the formation of conductive networks.

\section{Acknowledgements}

This work was supported by CONACyT (Mexico) Grant No. 79609 of Dr. F. Avilés. J. O. Aguilar is thankful to CONACyT for the granted postdoctoral fellowship at CICY. Raw PSF was kindly provided by 'Solvay Advanced Polymers', through Dr. Javier Leal. Technical assistance of Alejandro May (CICY) with sample preparation and Oscar Ceh (CINVESTAV) with electrical conductivity measurements is also strongly appreciated. We also thank Dr. Juan Cauich and Dr. Manuel Aguilar for their insightful comments.

\section{References}

[1] Li C., Thostenson E. T., Chou T. W.: Sensors and actuators based on carbon nanotubes and their composites: A review. Composites Science and Technology, 68, 1227-1249 (2008).

DOI: 10.1016/j.compscitech.2008.01.006

[2] Park C., Ounaies Z., Watson K. A., Pawlowski K., Lowther S. E., Connell J. W., Siochi E. J., Harrison J. S., St. Clair T. L.: Polymer single-walled carbon nanotube composites for potential aircraft applications. NASA ICASE report No. 2002-36, NASA/CR-2002211940 (2002).

[3] Abraham J. K., Philip B., Witchurch A., Varadan V. K., Redy C.C.: A compact wireless gas sensor using a carbon nanotube/PMMA thin film chemiresistor. Smart Materials and Structures, 13, 1045-1049 (2004). DOI: $10.1088 / 0964-1726 / 13 / 5 / 010$ 
[4] Curran S. A., Talla J., Dias S., Zhang D., Carroll D., Birx D.: Electrical transport measurements of highly conductive carbon nanotube/poly(bisphenol A carbonate) composite. Journal of Applied Physics, 105, 073711/1-073711/5 (2009).

DOI: $\underline{10.1063 / 1.3073938}$

[5] Singh I., Bhatnagar P. K., Mathur P. C., Kaur I., Bharadwaj L. M., Pandey R.: Optical and electrical characterization of conducting polymer-single walled carbon nanotube composite films. Carbon, 46, 11411144 (2008).

DOI: $10.1016 /$ j.carbon.2008.04.013

[6] Wang T., Lei C-H., Dalton A. B., Creton C., Lin Y., Fernando K. A. S., Sun Y-P., Manea M., Asua J. M., Keddie J. L.: Waterborne, nanocomposite pressuresensitive adhesives with high tack energy, optical transparency, and electrical conductivity. Advanced Materials, 18, 2730-2734 (2006).

DOI: $10.1002 / \mathrm{adma} .200601335$

[7] Grossiord N., Kivit P. J. J., Loos J., Meuldijk J., Kyrylyuk A. A., van der Schoot P., Koning C. E.: On the influence of the processing conditions on the performance of electrically conductive carbon nanotube/polymer nanocomposites. Polymer, 49, 2866-2872 (2008). DOI: $10.1016 /$ j.polymer.2008.04.033

[8] Peng H., Sun X.: Highly aligned carbon nanotube/ polymer composites with much improved electrical conductivities. Chemical Physics Letters, 471, 103105 (2009).

DOI: $10.1016 /$ j.cplett.2009.02.008

[9] Ma P. C., Tang B. Z., Kim J-K.: Effect of CNT decoration with silver nanoparticles on electrical conductivity of CNT-polymer composites. Carbon, 46, 14971505 (2008).

DOI: $10.1016 /$ j.carbon.2008.06.048

[10] Bauhofer W., Kovacs J. S.: A review and analysis of electrical percolation in carbon nanotube polymer composites. Composites Science and Technology, 69, 1486-1498 (2009).

DOI: 10.1016/j.compscitech.2008.06.018

[11] Li J., Ma P. C., Chow W. S., To C. K., Tang B. Z., Kim J-K.: Correlations between percolation threshold, dispersion state, and aspect ratio of carbon nanotubes. Advanced Functional Materials, 17, 3207-3215 (2007). DOI: $\underline{10.1002 / \mathrm{adfm} .200700065}$

[12] Xie X-L., Mai Y-W., Zhou X-P.: Dispersion and alignment of carbon nanotubes in polymer matrix: A review. Materials Science and Engineering R: Reports, 49, 89-112 (2005).

DOI: $10.1016 /$ j.mser.2005.04.002

[13] Song Y. S., Youn J. R.: Influence of dispersion states of carbon nanotubes on physical properties of epoxy nanocomposites. Carbon, 43, 1378-1385 (2005). DOI: $10.1016 /$ j.carbon.2005.01.007
[14] Martin C. A., Sandler J. K. W., Shaffer M. S. P., Schwarz M-K., Bauhofer W., Schulte K., Windle A. H.: Formation of percolating networks in multi-wall carbon-nanotube-epoxy composites. Composites Science and Technology, 64, 2309-2316 (2004).

DOI: $\underline{10.1016 / j . c o m p s c i t e c h .2004 .01 .025}$

[15] Seidel G. D., Boehringer K. L., Lagoudas D. C.: Analysis of clustering and interphase region effects on the electrical conductivity of carbon nanotube-polymer nanocompistes via computational micromechanics. in 'Proceedings of SMASIS 2008 Ellicott City, USA' p.7 (2008).

[16] Fu M., Yu Y., Xie J. J., Wang L. P., Fan M. Y., Liang S. L., Zeng Y. K.: Significant influence of film thickness on the percolation threshold of multiwall carbon nanotube/low density polyethylene composite films. Applied Physics Letters, 94, 012904/1-012904/3 (2009). DOI: $10.1063 / 1.3056055$

[17] Avilés F., Cauich-Rodríguez J. V., Moo-Tah L., MayPa A., Vargas-Coronado R.: Evaluation of mild acid oxidation treatments for MWCNT functional. Carbon, 47, 2970-2975 (2009).

DOI: $10.1016 /$ j.carbon.2009.06.044

[18] Liao Y-H., Marietta-Tondin O., Liang Z., Zhang C., Wang B.: Investigation of the dispersion process of SWNTs/SC-15 epoxy resin nanocomposites. Materials Science and Engineering A, 385, 175-181 (2004). DOI: $\underline{10.1016 / \mathrm{S} 0921-5093(04) 00857-3}$

[19] Hernández J. J., García-Gutiérrez M. C., Nogales A., Rueda D. R., Kwiatkowsa M., Szymczyk A., Roslaniecb Z., Conchesoc A., Guineac I., Ezquerra T. A.: Influence of preparation procedure on the conductivity and transparency of SWCNT-polymer nanocomposites. Composites Science and Technology, 69, 1867-1872 (2009). DOI: 10.1016/j.compscitech.2009.04.002

[20] Chang L., Friedrich K., Ye L., Toro P.: Evaluation and visualization of the percolating networks in multiwall carbon nanotube/epoxy composites. Journal of Materials Science, 44, 4003-4012 (2009). DOI: $10.1007 / \mathrm{s} 10853-009-3551-3$

[21] Sandler J. K. W., Kirk J. E., Kinloch I. A., Shaffer M. S. P., Windle A. H.: Ultra-low electrical percolation threshold in carbon-nanotube epoxy composites. Polymer, 44, 5893-5899 (2003). DOI: $10.1016 / \mathrm{S} 0032-3861(03) 00539-1$

[22] Kovacs J. Z., Velagala B. S., Schulte K., Bauhofer W.: Two percolation thresholds in carbon nanotube epoxy composites. Composites Science and Technology, 67, 922-928 (2007).

DOI: 10.1016/j.compscitech.2006.02.037

[23] Antonucci F., Faiella G., Giordano M., Nicolais L., Pepe G.: Electrical properties of single walled carbon nanotube reinforced polystyrene composites. Macromolecular Symposia, 247, 172-181 (2007).

DOI: $\underline{10.1002 / \text { masy. } 200750120}$ 
[24] Ha M. L. P., Grady B. P., Lolli G., Resasco D. E., Ford T. W.: Composites of single-walled carbon nanotubes and styrene-isoprene copolymer latices. Macromolecular Chemistry and Physics, 205, 446-456 (2007). DOI: $\underline{10.1002 / \mathrm{macp} .200600521}$

[25] Kymakis E., Alexandou I., Amaratunga G. A. J.: Single-walled carbon nanotube-polymer composites: Electrical, optical and structural investigation. Synthetic Metals, 127, 59-62 (2002). DOI: $10.1016 / \mathrm{S} 0379-6779(01) 00592-6$

[26] Du F., Guthy C., Kashiwagi T., Fischer J. E., Winey K. I.: An infiltration method for preparing single-wall nanotube/epoxy composites with improved thermal conductivity. Journal of Polymer Science B: Polymer Physics, 44, 1513-1519 (2006).

DOI: $10.1002 /$ polb.20801
[27] Seidel G. D., Lagoudas D. C.: A micromechanics model for the electrical conductivity of nanotubepolymer nanocomposites. Journal of Composite Materials, 43, 917-941 (2009).

DOI: $10.1177 / 0021998308105124$

[28] Xiao G., Tao Y., Lu J., Zhang Z.: Highly conductive and transparent carbon nanotube composite thin films deposited on polyethylene terephthalate solution dipping. Thin Solid Films, 518, 2822-2824 (2010). DOI: $10.1016 / j . t s f .2009 .11 .021$

[29] Blackburn J. L., Barnes T. M., Beard M. C., Kim YH., Tenent R. C., McDonald T. J., To B., Coutts T. J., Heben M. J.: Transparent conductive single-walled carbon nanotube networks with precisely tunable ratios of semiconducting and metallic nanotubes. ACS Nano, 2, 1266-1274 (2008).

DOI: $\underline{10.1021 / \mathrm{nn} 800200 \mathrm{~d}}$ 